\title{
EFEITO NEUROBIOLÓGICO DA PSICOTERAPIA: UMA REVISÃO SISTEMÁTICA
}

Yedda Jacqueline Almeida COUTO, Universidade de Rio Verde, jack_rv28@ hotmail.com. Claudio Herbert NINA-E-SILVA, Laboratório de Psicologia Anomalística e Neurociências, Universidade de Rio Verde. Lenny Francis Campos de ALVARENGA, Laboratório de Psicologia Anomalística e Neurociências, Universidade de Rio Verde.

\section{Recebido em: 30/10/2013 - Aprovado em: 20/12/2013 - Disponibilizado em: 15/01/2014}

Resumo: O presente trabalho de revisão sistemática da literatura teve como objetivo verificar se há relação entre a psicoterapia e a plasticidade cerebral. Foram analisados 35 artigos de periódicos internacionais cuja data de publicação variasse entre 2001 e 2012. Em cada um dos artigos revisados, buscou-se identificar o tipo de psicoterapia, o tipo de transtorno psicológico e os efeitos dessa psicoterapia sobre estruturas e funções cerebrais. Verificou-se que a psicoterapia tem um efeito na minimização dos transtornos psicológicos devido à sua capacidade de promover uma transformação e recondicionamento no funcionamento da atividade neural do sujeito. Nos artigos revisados, observouse evidências empíricas de que a psicoterapia altera o funcionamento de áreas especificas do cérebro através da alteração dos padrões de comunicação neural. Portanto, concluiu-se que a literatura dá suporte empírico para a afirmação de que há relação entre a psicoterapia e a plasticidade cerebral.

Palavras-chave: Psicoterapia, Plasticidade Cerebral, Psicopatologia, Neufisiologia.

Abstract: This systematic review of the literature aimed to verify if there is a relationship between psychotherapy and brain plasticity. We analyzed 35 articles published in international journals whose publication date varied between 2001 and 2012. In each of the papers, we sought to identify the type of psychotherapy, the kind of psychological disorder and the effects of psychotherapy on brain structures and functions. It was found that psychotherapy has an effect on minimizing psychological problems because of their ability to promote processing and reconditioning operation of the neural activity in the subject. In the articles reviewed, we found empirical evidence that psychotherapy alters the functioning of specific areas of the brain by altering patterns of neural communication. Therefore, it was concluded that the literature provides empirical support for the claim that there is a relationship between psychotherapy and brain plasticity ..

Keywords:Psychotherapy, Brain Plasticity, Psychopathology, Neurophisiology.

\section{Introdução}

O sistema nervoso é capaz de se moldar às experiências. Esse conceito revolucionário, ainda ignorado por muitos psicólogos, tem sido demonstrado empiricamente apenas nas últimas duas décadas (NORTHOFF et al., 2007; LINDEN, 2008; KONARSKI, et al., 2009; DOIDGE, 2011; BOEKER et al., 2013). A capacidade de adaptação do sistema nervoso às influências ambientais ocorre devido à sua natureza plástica e mutável (BORGERTS, 1996; KANDEL, 1999; BEAUREGARD, 2007; BEAUREGARD, 2009).
Atualmente, o avanço das pesquisas nas neurociências tem evidenciado que desenvolvimento neural depende de interação social (ETKIN, PITTENGER \& KANDEL, 2005; MUNDO, 2006; BOEKER et al., 2013). Sendo assim, ao contrário do que se imaginava até há pouco tempo, o sistema nervoso está propicio a adaptar-se a mudanças nas relações sociais, novas possibilidades de vida e trabalho, rotinas e limitações (LINDEN, 2008; BEAUREGARD, 2007; BEAUREGARD, 2009; DOIDGE, 2011).

Dessa maneira, o psicólogo clínico deve ter consciência de que a psicoterapia tem um efeito na atividade mental e no comportamento do paciente devido à sua 
capacidade de promover uma transformação e recondicionamento no funcionamento da atividade neural desse indivíduo (BOEKER et al., 2013).

Sintetizando, a psicoterapia nos traz a possibilidade de alterar comportamentosproblema por meio da modificação do funcionamento e da estrutura de áreas especificas do cérebro (KANDEL, 1979; BAXTER et al., 1992; BORGERTS, 1996; ETKIN, PITTENGER \& KANDEL, 2005; ETKIN, PITTENGER, POLAN \& KANDEL, 2005; MUNDO, 2006; DOIDGE, 2011; BOEKER et al., 2013).

Por isso, o presente trabalho teve como objetivo revisar os trabalhos científicos mais relevantes sobre os efeitos da psicoterapia sobre o sistema nervoso, descrevendo os efeitos da psicoterapia sobre a estrutura e a função do sistema nervoso.

\section{Materiais e Métodos}

O presente estudo foi uma pesquisa bibliográfica, de natureza quantitativa, constituindo-se em uma revisão sistemática da literatura. Para tanto, consultou-se a biblioteca virtual PubMed (United States National Library of Medicine). Os termos de busca utilizados foram "psychotherapy brain changes".

Para a composição da amostra de consulta, foram arbitrados os seguintes critérios de inclusão: 1) artigos publicados apenas no século XXI (a partir de 2001); 2) artigos publicados em periódicos com corpo editorial (avaliação por pares) e circulação internacional.

A partir da lista de artigos fornecidos pela PubMed em resposta aos termos de busca, procedeu-se à seleção daqueles artigos que obedecessem aos critérios de inclusão na amostra. Os artigos assim selecionados foram copiados do banco de artigos da PubMed, em formato digital PDF, para uma pasta específica de computador portátil. Em seguida, cada um dos artigos foi lido com o objetivo de se registrar as seguintes categorias de análise: tipo de psicoterapia, tipo de transtorno psicológico, área cerebral modificada, tipo de modificação da área cerebral.

\section{Resultados e Discussão}

Utilizando-se os termos de busca e os critérios de inclusão descritos no Método, obteve-se uma amostra de 35 artigos sobre os efeitos neurobiológicos da psicoterapia. Foram observados quatro tipos de psicoterapia na amostra: psicoterapia cognitivo-comportamental, psicoterapia comportamental, psicoterapia psicodinâmica e psicoterapia interpessoal.

A psicoterapia cognitiva comportamental (TCC) foi o tipo de psicoterapia mais frequentemente observada na amostra investigada, com dezesseis trabalhos revisados (vide Quadro 1). Essa modalidade de psicoterapia apresentou eficácia do seu modo intervenção nos seguintes transtornos: episódio depressivo 
maior, esquizofrenia, ansiedade, TOC (transtorno obsessivo-compulsivo).

De modo geral, os resultados positivos da TCC estiveram relacionados ao restabelecimento do controle da atenção, a reestruturação da memória em curto prazo e a ressignificação do pensamento por meio do aprendizado.

Essas modificações psicológicas promovidas pela TCC foram relacionadas à alteração da atividade metabólica neuronal nas seguintes áreas cerebrais: giro do cíngulo, córtex frontal (incluindo o motor).

Quadro 1 - Descrição dos efeitos neurobiológicos da psicoterapia cognitivo-comportamental categorizados por tipo de transtorno.

\begin{tabular}{|c|c|c|c|}
\hline Transtorno & Área Cerebral & Efeito & Referência \\
\hline \multirow[t]{2}{*}{$\begin{array}{l}\text { Obsessivo- } \\
\text { Compulsivo }\end{array}$} & Núcleo Caudado Direito & Redução do Metabolismo & $\begin{array}{l}\text { Linden (2006), } \\
\text { Linden (2008) }\end{array}$ \\
\hline & Córtex Frontal (Córtex Motor) & Inibição cortical & Radhu et al. (2012) \\
\hline \multirow[t]{2}{*}{ Fobia específica } & Límbica e Paralímbica & Redução da Atividade & Linden (2006) \\
\hline & $\begin{array}{l}\text { Giro do Cíngulo } \\
\text { Cortex Insular }\end{array}$ & Ativação metabólica & Straube et al. (2006) \\
\hline Fobia Animal & $\begin{array}{l}\text { Córtex Pré frontal } \\
\text { Giro parahipocampal }\end{array}$ & $\begin{array}{l}\text { Ativação da atividade } \\
\text { metabólica }\end{array}$ & Paquette et al. (2003) \\
\hline Fobia social & Córtex Frontal & Redução da atividade EEG & Moscovitch et al. (2011) \\
\hline \multirow[t]{2}{*}{ Ansiedade } & Giro do cíngulo & $\begin{array}{l}\text { Alteração morfológica } \\
\text { Ativação metabólica }\end{array}$ & Quidé et al. (2012) \\
\hline & $\begin{array}{l}\text { Hipocampo } \\
\text { Amigdala } \\
\text { Córtex Pré-frontal }\end{array}$ & $\begin{array}{l}\text { Redução da atividade } \\
\text { metabólica }\end{array}$ & Rüegg (2004) \\
\hline $\begin{array}{l}\text { Estresse Pós- } \\
\text { Traumático }\end{array}$ & Córtex Frontal & Redução da atividade EEG & Rabe et al. (2008) \\
\hline Fibromialgia & Córtex Pré-frontal ventrolateral & Ativação Metabólica & Jensen et al. (2012) \\
\hline \multirow[t]{2}{*}{ Esquizofrenia } & $\begin{array}{l}\text { Córtex frontal } \\
\text { Córtex insular } \\
\text { Tálamo } \\
\text { Putamen }\end{array}$ & $\begin{array}{l}\text { Redução da atividade } \\
\text { metabólica }\end{array}$ & Kumari et al. (2011) \\
\hline & $\begin{array}{l}\text { Lobo frontal } \\
\text { Giro Pré-central } \\
\text { Lobo parietal inferior direito } \\
\text { Giro temporal superior }\end{array}$ & Aumento do volume & Premkumar et al. (2009) \\
\hline \multirow[t]{7}{*}{$\begin{array}{l}\text { Episódio } \\
\text { depressivo maior }\end{array}$} & Córtex Pré-frontal & Redução do Metabolismo & $\begin{array}{l}\text { Linden (2006), } \\
\text { Linden (2008) }\end{array}$ \\
\hline & $\begin{array}{l}\text { Giro do Cíngulo } \\
\text { Hipo campo }\end{array}$ & $\begin{array}{l}\text { Aumento da atividade } \\
\text { metabólica }\end{array}$ & \multirow[t]{2}{*}{ Goldapple et al. (2004) } \\
\hline & Córtex Frontal & $\begin{array}{l}\text { Redução da atividade } \\
\text { metabólica }\end{array}$ & \\
\hline & $\begin{array}{l}\text { Córtex Órbito-Frontal } \\
\text { Córtex pré frontal } \\
\text { Giro do Cíngulo }\end{array}$ & $\begin{array}{l}\text { Redução da atividade } \\
\text { metabólica }\end{array}$ & Keneddy et al. (2007) \\
\hline & $\begin{array}{l}\text { Eixo HPA } \\
\text { Córtex Pré-Frontal }\end{array}$ & $\begin{array}{l}\text { Alteração Morfológica } \\
\text { Redução da atividade } \\
\text { elétrica }\end{array}$ & Sharpley (2010) \\
\hline & Córtex pré-frontal & $\begin{array}{l}\text { Redução da atividade } \\
\text { metabólica }\end{array}$ & \multirow[t]{2}{*}{ Goldapple et al. (2004) } \\
\hline & $\begin{array}{l}\text { Hipocampo } \\
\text { Giro do Cíngulo }\end{array}$ & $\begin{array}{l}\text { Aumento da atividade } \\
\text { metabólica }\end{array}$ & \\
\hline
\end{tabular}

Fonte: Os autores.

A psicoterapia comportamental foi $\mathrm{o}$ segundo tipo mais freqüente de psicoterapia observado na amostra, com seis artigos

(Quadro 2). A psicoterapia comportamental 
apresentou resultados positivos na modificação dos comportamentos-problema presentes nos seguintes transtornos: ansiedade, esquizofrenia, fobia especifica e TOC (transtorno obsessivo compulsivo).

Esses resultados positivos foram relacionados à modificação, pela psicoterapia comportamental, das seguintes áreas

Quadro 2 - Descrição dos efeitos neurobiológicos da psicoterapia comportamental categorizados por tipo de transtorno.

\begin{tabular}{|l|l|l|l|}
\hline Transtorno & Área Cerebral & Efeito & Referência \\
\hline Fobia & Paralímbica & Redução da Atividade & Linden (2008) \\
\hline Ansiedade & Redução da Atividade & Roffman et al. (2005) \\
\hline Ansiedade & $\begin{array}{l}\text { Giro do Cíngulo } \\
\text { Córtex Pré-frontal } \\
\text { Córtex insular }\end{array}$ & Ativac̃o metabólica & $\begin{array}{l}\text { Frewen, Dozois \& Lanius } \\
(2007)\end{array}$ \\
\hline Ansiedade & Sistema Límbico & $\begin{array}{l}\text { Redução da atividade } \\
\text { metabólica }\end{array}$ & Roffman et al (2005) \\
\hline Esquizofrenia & Córtex pré-frontal & $\begin{array}{l}\text { Aumento da atividade } \\
\text { metabólica }\end{array}$ & Kumari et al (2009) \\
\hline TOC & Fluxo sanguíneo regional cerebral & Aumento da atividade & Nakatania et al (2003) \\
\hline
\end{tabular}

Fonte: Os autores.

Quadro 3 - Descrição dos efeitos neurobiológicos da psicoterapia psicodinâmica categorizados por tipo de transtorno.

\begin{tabular}{|l|l|l|l|}
\hline Transtorno & Área Cerebral & Efeito & Referência \\
\hline Pânico & $\begin{array}{l}\text { Hipocampo } \\
\text { Amigdala } \\
\text { Córtex Pré-frontal }\end{array}$ & $\begin{array}{l}\text { Redução da atividade } \\
\text { metabólica }\end{array}$ & $\begin{array}{l}\text { Beutel (2006) } \\
\text { Beutel } \text { et al (2010) }\end{array}$ \\
\hline $\begin{array}{l}\text { Episódio depressivo } \\
\text { maior }\end{array}$ & $\begin{array}{l}\text { Transportador da serotonina } \\
\text { (mesencéfalo) } \\
\text { Transportadores da } \\
\text { dopamina (estriato) }\end{array}$ & Aumento do volume & Letho et al (2008) \\
\hline $\begin{array}{l}\text { Episódio depressivo } \\
\text { maior }\end{array}$ & $\begin{array}{l}\text { Hipocampo } \\
\text { Amigdala } \\
\text { Giro do Cingulo } \\
\text { Córtex Pré-frontal }\end{array}$ & $\begin{array}{l}\text { Redução da atividade } \\
\text { metabólica }\end{array}$ & Buchheim et al (2012) \\
\hline $\begin{array}{l}\text { Ansiedade } \\
\text { Generalizada }\end{array}$ & $\begin{array}{l}\text { Amigdala } \\
\text { Córtex Pré-frontal }\end{array}$ & $\begin{array}{l}\text { Redução da atividade } \\
\text { metabólica }\end{array}$ & Whitehead (2006) \\
\hline
\end{tabular}

Fonte: Os autores.

Os efeitos específicos da psicoterapia comportamental nessas áreas foram basicamente devido à alteração na atividade metabólica neuronal.

$$
\text { A psicoterapia psicanalítica foi } o
$$
terceiro tipo de psicoterapia mais registrado na amostra, com cinco trabalhos (Quadro 3). tratamento dos seguintes transtornos: episodio depressivo maior, ansiedade generalizada e pânico.

De acordo com a análise da amostra, as áreas modificadas pela psicoterapia psicanalítica foram: o hipocampo, amígdala, córtex pré-frontal, transportador de serotonina

A psicoterapia psicanalítica foi eficaz no 
(mesencéfalo), transportadores de dopamina (estriato), giro do cíngulo.

Os principais efeitos promovidos pela psicoterapia psicanalítica nas áreas citadas foram a redução da atividade metabólica e o aumento de volume (densidade). Esses resultados estão de acordo com a literatura
(BEUTEL et al., 2006; BOEKER et al., 2013) segundo a qual a psicofarmacoterapia e a psicoterapia de base psicanalítica produzem alterações similares na atividade neural, embora por meio de mecanismo específicos bastante distintos.

Quadro 4 - Descrição dos efeitos neurobiológicos da psicoterapia cognitiva categorizados por tipo de transtorno.

\begin{tabular}{|l|l|l|l|}
\hline Transtorno & Área Cerebral & Efeito & Referência \\
\hline $\begin{array}{l}\text { Déficit Cognitivo } \\
\text { Leve }\end{array}$ & Hipocampo & $\begin{array}{l}\text { Aumento da atividade } \\
\text { metabólica }\end{array}$ & Rosen et al (2011) \\
\hline Esquizofrenia & $\begin{array}{l}\text { Córtex Pré-frontal } \\
\text { Giro do Cíngulo }\end{array}$ & $\begin{array}{l}\text { Aumento da atividade } \\
\text { metabólica }\end{array}$ & Haut et al (2010) \\
\hline $\begin{array}{l}\text { Estresse pós } \\
\text { traumático }\end{array}$ & $\begin{array}{l}\text { Parietal } \\
\text { Occipital }\end{array}$ & $\begin{array}{l}\text { Aumento da atividade } \\
\text { metabólica }\end{array}$ & Adenauer et al (2011) \\
\hline Estresse agudo & Hipocampo & Aumento do volume & Davidson \& McEwen (2012) \\
\hline $\begin{array}{l}\text { Episódio depressivo } \\
\text { maior }\end{array}$ & Córtex Pré-frontal & $\begin{array}{l}\text { Redução da atividade } \\
\text { metabólica }\end{array}$ & $\begin{array}{l}\text { De Rubeis, Siegel \& Hollon } \\
(2008)\end{array}$ \\
\hline
\end{tabular}

Fonte: Os autores.

Quadro - 5: Descrição dos efeitos neurobiológicos da psicoterapia Interpessoal categorizados por tipo de transtorno.

\begin{tabular}{|l|l|l|l|}
\hline Transtorno & Área Cerebral & Efeito & Referência \\
\hline \multirow{2}{*}{$\begin{array}{l}\text { Episódio depressivo } \\
\text { maior }\end{array}$} & $\begin{array}{l}\text { Sistema límbico } \\
\text { Giro do Síngulo }\end{array}$ & $\begin{array}{l}\text { Ativação do fluxo } \\
\text { sanguíneo }\end{array}$ & Martin et al (2001) \\
\cline { 2 - 3 } & $\begin{array}{l}\text { Eixo HPA } \\
\text { Córtex Pré-Frontal }\end{array}$ & $\begin{array}{l}\text { Alteração Morfológica } \\
\text { Redução da atividade } \\
\text { elétrica }\end{array}$ & Sharpley (2010) \\
\cline { 2 - 3 } & $\begin{array}{l}\text { Córtex Pré-Frontal, caudado } \\
\text { e tálamo }\end{array}$ & $\begin{array}{l}\text { Redução da atividade } \\
\text { metabólica }\end{array}$ & Brody et al (2001) \\
\cline { 2 - 3 } & Lobo Temporal & $\begin{array}{l}\text { Aumento da atividade } \\
\text { metabólica }\end{array}$ & \\
\hline
\end{tabular}

Fonte: Os autores.

A psicoterapia cognitiva, assim como a psicoterapia psicanalítica, foi o terceiro tipo mais freqüente de psicoterapia observado na amostra de cinco artigos (Quadro 4). A abordagem da psicoterapia cognitiva apresentou resultados positivos na mudança e ou reestruturação de pensamentos disfuncionais e esquemas inadequados nos seguintes transtornos: esquizofrenia, estresse pós traumático, estresse agudo e déficit cognitivo leve.
As principais áreas que sofreram modificações pela psicoterapia cognitiva foram: o hipocampo, o córtex pré-frontal, o giro do cíngulo, córtices parietal e occipital. Assim como a terapia comportamental, a psicoterapia cognitiva obteve os efeitos específicos de alteração na atividade metabólica neuronal das áreas cerebrais citadas.

A psicoterapia interpessoal foi o tipo de psicoterapia menos frequente na amostra estudada, com três casos descritos (Quadro 5).

A terapia interpessoal evidenciou eficácia na 
remissão dos sintomas de episódio depressivo maior.

As áreas cerebrais alteradas pela psicoterapia interpessoal foram: o sistema límbico, giro cíngulo, eixo HipotálamoHipófise-Adrenais (HPA), córtex pré-frontal, núcleo caudado, tálamo, lobo temporal. A psicoterapia interpessoal modificou as áreas cerebrais citadas em termos de ativação do fluxo sanguíneo regional, alteração morfológica, redução da atividade elétrica, redução da atividade metabólica e aumento da atividade metabólica.

A partir da análise da amostra, pôde-se perceber que a psicoterapia tem um papel fundamental para se trabalhar com plasticidade cerebral, promovendo resultados surpreendentes tanto quanto com os da psicofarmacologia. Com apenas uma ressalva, onde a psicoterapia modifica a estrutura e não apenas a função, promovendo desta forma um resultado mais duradouro (LINDEN, 2008; BEAUREGARD, 2007; BEAUREGARD, 2009; BOEKER et al., 2013).

Um resultado interessante foi o fato de que todas as quatro abordagens de psicoterapias registradas na amostra promoveram alterações cerebrais. Esse fato indica que, a despeito de sua base epistemológica, a psicoterapia, quando conduzida adequadamente, promove mudanças no sistema nervoso que permitem que se alcance, então, a melhora psicológica desejada.
Desse modo, os dados do presente estudo permitem afirmar que, seja qual for abordagem psicoterápica, as modificações cerebrais e as respectivas alterações psicológicas acontecerão respeitando os limites de cada cliente/paciente.

\section{Conclusão}

Por meio de uma revisão sistemática da literatura empírica recente, o presente estudo demonstrou que a base neurobiológica da psicoterapia reside na alteração dos padrões de comunicação neural. Essa alteração na comunicação neural ocorre pelas duas formas básicas de modificação cerebral pela psicoterapia descrita na literatura revisada: alteração metabólica e modificação estrutural.

Além disso, a psicoterapia pode trazer mudanças semelhantes aos que medicamentos psicofármacos promovem no sistema nervoso, alterando de forma semelhante a comunicação neural, e essas modificações podem ser comprovados a partir de exames de neuroimagem.

Portanto, os resultados deste estudo permitem afirmar que não existe atividade psicológica independente de uma atividade biológica subjacente. Dessa maneira, toda prática psicológica é, na verdade, uma prática biológica, tornando irrelevante a discussão sobre uma suposta dicotomia biologiaaprendizagem na Psicologia.

\section{Referências Bibliográficas}


BAXTER, L.R.; SCHWARTZ, J.M.; BERGMAN K.S.; SZUBA, M.P.; GUZE, B.H.; MAZZIOTTA, J.C.; ALAZRAKI, A.; SELIN, C.E.; FERNG, H. K.; MUNFORD, P. Caudate glucose metabolic rate changes with both drug and behavior therapy for obsessivecompulsive disorder. Archives of General Psychiatry, 49, 681-689, 1992.

BEAUREGARD, M. Effect of mind on brain activity: evidence from neuroimaging studies of psychotherapy and placebo effect. Nordic Journal of Psychiatry, 63(1), 5-16, 2009.

BEAUREGARD, M. Mind does really matter: evidence from neuroimaging studies of emotional self-regulation, psychotherapy, and placebo effect. Progress in Neurobiology, 81(4), 218-236, 2007

BEUTEL, M.E. Functional neuroimaging in psychotherapy research.

Bundesgesundheitsblatt

Gesundheitsforschung Gesundheitsschutz, 49(8), 749-58, 2006.

Borgerts, B. Plasticity of brain structure and function as the neurobiological principle of psychotherapy. Zeitchrifts for Klinische Psychologie Psychiatrie und Psychotherapie, 23, 124-131, 1996

BOEKER, H.; RICHTER, A.; HIMMIGHOFFEN, H.; ERNST, J.; BOHLEBER, L.; HOFMANN, E.; VETTER, J.; NORTHOFF, G. Essentials of psychoanalytic process and change: how can we investigate the neural effects of psychodynamic psychotherapy in individualized neuro-imaging? Frontiers in Human Neuroscience, 7, 355-369, 2013.

DOIDGE, N. (2011). O cérebro que se transforma. São Paulo: Record.

ETKIN, A.; PITTENGER, C.; KANDEL, E. Biology in the Service of Psychotherapy. Em J. Oldham, A. Skodol \& D. Bender (Orgs), Textbook of Personality Disorders (p. 669682). Nova Iorque: American Psychiatric Publishing, 2005.
ETKIN, A.; PITTENGER, C.; POLAN, H.J.; KANDEL, E.R. Toward a neurobiology of psychotherapy: basic science and clinical applications. The Journal of Neuropsychiatry and Clinical Neuroscience, 17(2), 145-58. 2005

KANDEL E.R. Psychotherapy and the single synapse: the impact of psychiatric thought on neurobiologic research. The New England Journal of Medicine, 301: 1028-1037, 1979.

KANDEL, E. R. Biology and the future of psychoanalysis: A new intellectual framework for psychiatry revisited. American Journal of Psychiatry, 156, 505-524, 1999.

KONARSKI J. K.; KENNEDY, S.H.; SEGAL, Z.V.;. LAU, M.A.; BIELING, P.J.; MCINTYRE, R.S.; MAYBERG, H.S. Predictors of nonresponse to cognitive behavioural therapy or venlafaxine using glucose metabolism in major depressive disorder. Journal of Psychiatry Neuroscience. 34(3), 175-180;

LINDEN, D.E. Brain imaging and psychotherapy: methodological considerations and practical implications. European Archive of Psychiatry Clinical Neuroscience, 258 (5), 71-75, 2008.

MUNDO, E. Neurobiology of dynamic psychotherapy: an integration possible? The Journal of the American Academy of Psychoanalysis and Dynamic Psychiatry, 34(4), 679-691.

NORTHOFF, G.; BERMPOHL, F.; SCHOENEICH, F.; BOEKER, H. How does our brain constitute defense mechanisms? First-person, neuroscience and psychoanalysis. Psychoterapy and Psychosomatics, 76(3), 141-153. 